\title{
3D Ultrasound-based Patient Positioning for Radiotherapy
}

\author{
Michael H. Wang ${ }^{a}$, Robert N. Rohling ${ }^{a}$, Neculai Archip ${ }^{b}$ and Brenda G. Clark ${ }^{c}$ \\ ${ }^{a}$ Department of Electrical and Computer Engineering, University of British Columbia, \\ Vancouver, B.C., Canada \\ ${ }^{b}$ Harvard Medical School, Brigham and Womens Hospital, Boston, MA, USA \\ ${ }^{c}$ The Ottawa Hospital Regional Cancer Center, Ottawa, Ontario, Canada
}

\begin{abstract}
A new 3D ultrasound-based patient positioning system for target localisation during radiotherapy is described. Our system incorporates the use of tracked 3D ultrasound scans of the target anatomy acquired using a dedicated $3 \mathrm{D}$ ultrasound probe during both the simulation and treatment sessions, fully automatic 3D ultrasound-toultrasound registration, and OPTOTRAK IRLEDs for registering simulation CT to ultrasound data. The accuracy of the entire radiotherapy treatment process resulting from the use of our system, from simulation to the delivery of radiation, has been validated on a phantom. The overall positioning error is less than $5 m m$, which includes errors from estimation of the irradiated region location in the phantom.
\end{abstract}

Keywords: Image-Guided Radiation Therapy, Image to Physical Space Registration, Localization, Ultrasound Guidance

\section{INTRODUCTION}

The accuracy of radiotherapy treatment is limited by how precisely the patient's position during the simulation procedure can be repeated in every treatment fraction. It is well known that patient movement, inaccurate set-up, or organ motion can induce positioning errors. ${ }^{1}$ These errors cause the dose distribution received by the patient to be different from that which is planned, potentially compromising the clinical outcome of the treatment.

Recently, the commercial systems BAT (North American Scientific Corp, Chatsworth, CA) and SonArray (Zmed Inc, Ashland, MA), which use ultrasound to verify the position of the tumour prior to the commencement of daily treatment, have been receiving considerable attention. ${ }^{2-7}$ In addition, the Restitu system (Resonant Medical Inc., Montreal, Canada) has been approved for marketing by the FDA. ${ }^{8}$ These systems offer a substantial improvement in patient localisation accuracy. However, they still have a number of limitations, such as requiring significant operator involvement in determining positional offsets, being susceptible to imaging errors, ${ }^{4,9}$ and having reported mean inter-operator variabilities of up to $7 \mathrm{~mm}$ in a single direction. ${ }^{5}$

This paper proposes a new approach for patient positioning in radiotherapy using ultrasound with potentially improved accuracy, ease of use, and flexibility. The system uses spatially registered simulation CT and ultrasound data with ultrasound images taken immediately prior to treatment to determine the exact target isocenter location in the coordinates of the linear accelerator (LINAC). A 3D ultrasound machine with dedicated volumetric probes will be used to acquire patient ultrasound data. The system will also utilise fully automatic 3D image registration techniques to compare simulation and treatment ultrasound images of the patient.

The objectives of this paper are to describe the new proposed ultrasound-based patient positioning system and to present preliminary experimental results of its accuracy in positioning a radiosensitive phantom for irradiation by a LINAC. Previously, little work has been done to assess the accuracy of ultrasound guidance systems for radiotherapy. ${ }^{7}$ Our experimental methods allow the location of the final delivered dose to be compared to the planned dose, thereby closing the loop on the entire treatment process using our system. No previous work has shown this type of validation.

Further author information: (Send correspondence to M.H.W.) M.H.W.: E-mail: mikew@ece.ubc.ca

Medical Imaging 2006: Visualization, Image-Guided Procedures, and Display,

edited by Kevin R. Cleary, Robert L. Galloway, Jr., Proc. of SPIE Vol. 6141, 61411K, (2006) 1605-7422/06/\$15 - doi: 10.1117/12.653225

Proc. of SPIE Vol. $614161411 \mathrm{~K}-1$ 


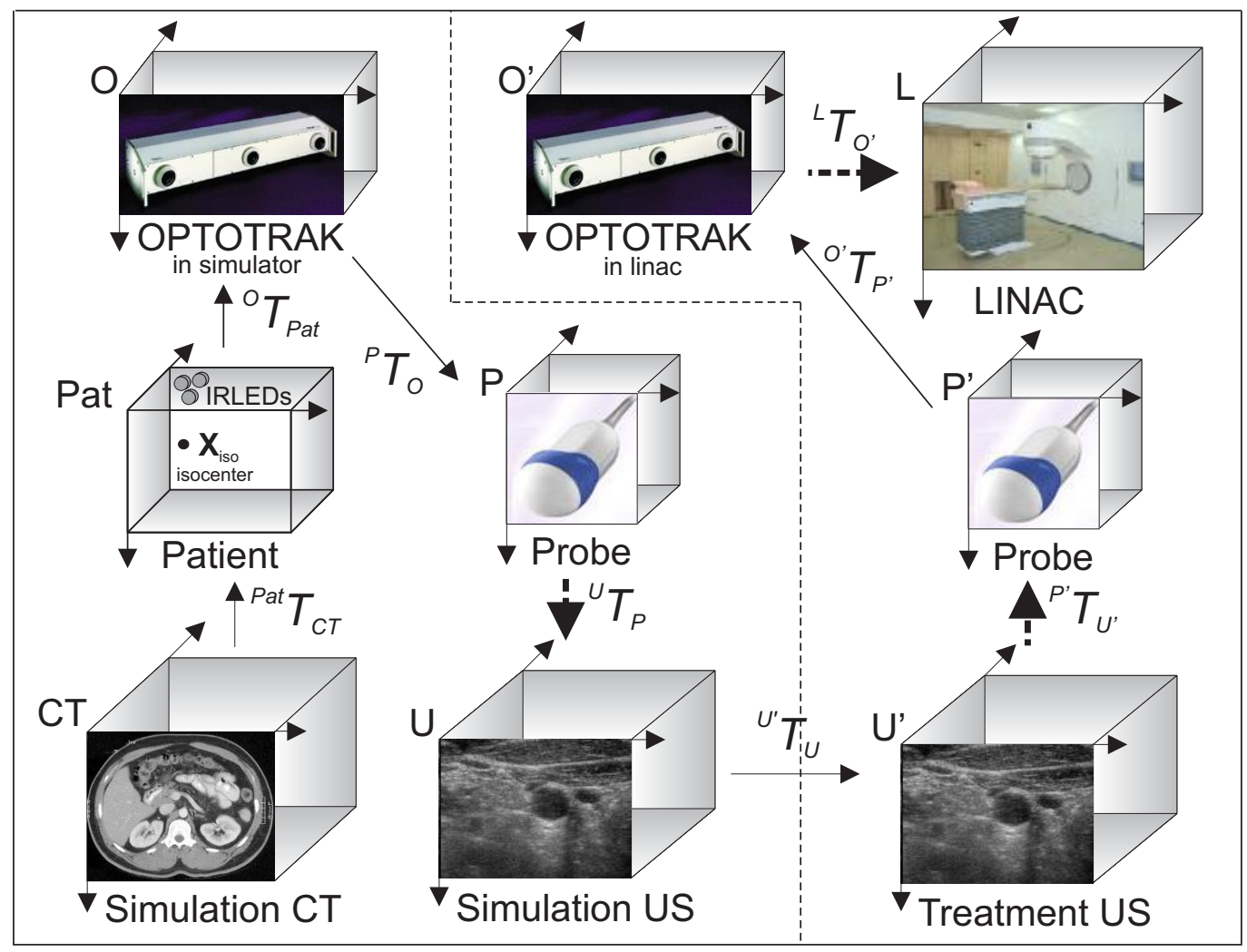

Figure 1. Coordinate transformations involved to locate the treatment isocenter in the LINAC coordinates using the proposed system. Calibration parameters known a priori are shown by dashed arrows. The data collected during the simulation procedure and before each treatment fraction are shown in the left and right panels, respectively.

\section{SYSTEM OVERVIEW}

The envisaged radiotherapy treatment protocol using the proposed ultrasound patient positioning system is as follows. During the CT simulation procedure, Optotrak (NDI, Waterloo, Canada) infra-red LEDs (IRLEDs) are placed on the patient's skin before the commencement of the CT scan so that they appear in the CT image as fiducial markers. Immediately after the scan, simulation ultrasound volumes of relevant anatomy near the target isocenter are acquired. During this procedure, both the ultrasound probe and patient are tracked by the OPTOTRAK using IRLEDs attached to the probe and patient. The acquired data is then processed offline, and the CT and ultrasound volumes registered. In the treatment session, the patient is set up as usual using skin marks and alignment lasers. Due to possible errors, such as target motion with respect to the skin markers, the target may not be exactly at the expected location. A treatment ultrasound volume of the target anatomy is then taken with a tracked probe to measure the true target location. The treatment ultrasound is then automatically registered to the simulation ultrasound data, allowing the planned dose geometry defined in the simulation CT scan to be transformed into the LINAC coordinate system.

The series of coordinate transformations that are used by the proposed system to localise the treatment isocenter inside the LINAC are shown in Fig. 1 and summarised below:

${ }^{\mathbf{C}} \mathbf{x}_{\text {iso }}$ : This is the planned treatment isocenter on the CT scan determined by the radiation oncologist.

${ }^{\text {Pat }} \mathbf{T}_{\mathbf{C T}}$ : The CT to patient transformation may be calculated by using the IRLED locations on the patient's skin and in the CT scan to form a set of corresponding points in both coordinates.

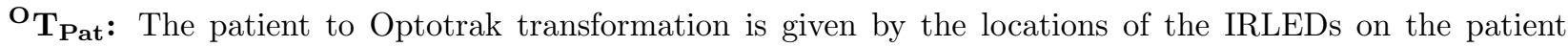
measured by Optotrak. 
${ }^{\mathbf{P}_{\mathbf{O}}}$ : The Optotrak to ultrasound probe transformation is based on Optotrak measured locations of the IRLEDs on the probe.

$\mathbf{U}_{\mathbf{P}}$ : The ultrasound probe to ultrasound image transformation is constant and is pre-determined by an off-line calibration procedure.

$\mathbf{U}^{\prime} \mathbf{T}_{\mathbf{U}}$ : The transformation between simulation and treatment ultrasound images of the target anatomy is calculated using a suitable automatic registration algorithm.

$\mathbf{P}^{\prime} \mathbf{T}_{\mathbf{U}^{\prime}}$ : The ultrasound probe calibration parameters for the treatment session.

$\mathbf{O}^{\prime} \mathbf{T}_{\mathbf{P}^{\prime}}$ : The ultrasound probe to Optotrak transformation is again given by Optotrak measurements.

${ }^{\mathbf{L}} \mathbf{T}_{\mathbf{O}^{\prime}}$ : The Optotrak to LINAC transformation is pre-determined using an off-line calibration procedure.

The matrix $\mathbf{T}$ represents a homogeneous transformation including both orientation and position. The entire chain of transformations required to find the treatment isocenter location in the LINAC coordinate system $\left({ }^{\mathbf{L}} \mathbf{x}_{\mathbf{i s o}}\right)$ prior to irradiation is given by:

$$
{ }^{\mathbf{L}} \mathbf{X}_{\text {iso }}={ }^{\mathbf{L}} \mathbf{T}_{\mathbf{O}^{\prime}}{ }^{\mathbf{O}^{\prime}} \mathbf{T}_{\mathbf{P}^{\prime}}{ }^{\mathbf{P}^{\prime}} \mathbf{T}_{\mathbf{U}^{\prime}}{ }^{\mathbf{U}^{\prime}} \mathbf{T}_{\mathbf{U}}{ }^{\mathbf{U}_{\mathbf{P}}} \mathbf{T}_{\mathbf{P}}{ }^{\mathbf{P}} \mathbf{T}_{\mathbf{O}}{ }^{\mathbf{O}} \mathbf{T}_{\mathbf{P a t}}{ }^{{ }^{\mathrm{Pat}}} \mathbf{T}_{\mathbf{C T}}{ }^{\mathrm{C} \mathbf{T}_{\mathbf{X}}} \mathbf{x}_{\text {iso }}
$$

Similar to the Restitu, our system performs intra-modality ultrasound-to-ultrasound registration to compare the patient anatomy at the time of treatment to its location during simulation. In contrast, the BAT and SonArray systems require inter-modality CT to ultrasound registration to be performed manually by eye. Our method of conducting inter-modality ultrasound registration has a number of advantages. Firstly, the accuracy of the registration is not dependant on operator skill. In addition, anatomical features in CT and ultrasound images can have different appearances, making accurate alignment difficult and subjective to interpretation. ${ }^{5}$ Finally, the effect of systematic ultrasound imaging errors on registration accuracy, which could include refraction, speed of sound artefacts, probe calibration error, and possible tissue deformation due to probe pressure, may be reduced when comparing ultrasound images acquired in a repeatable manner using the same imaging parameters, instead of ultrasound to CT images.

Another difference between our system and all the existing alternatives is that dedicated 3D ultrasound probes are used to acquire ultrasound images. This removes the need for the operator to sweep a 2D probe across the patient's skin, further promoting the reproducibility of ultrasound images used for registration. In addition, the use of dedicated 3D ultrasound probes allows 3D images with isotropic sampling and parallel slices to be created, which facilitates the use of automatic pixel intensity based image registration in our system. In contrast, anatomical features in other systems are normally segmented manually either in CT or ultrasound to enable registration to be performed.

Finally, one more difference between the proposed system and the Restitu lies in the method used to register simulation CT and ultrasound scans. In acquiring the simulation ultrasound, IRLEDs are used to track both the patient and the ultrasound probe simultaneously by the proposed system. The IRLED locations recorded on the patient and in the simulation CT are used to register the simulation CT and ultrasound. Therefore, patient motion between the acquisition of these two scans can be detected, allowing the rigid-body component of that motion to be corrected. In contrast, the Restitu system assumes that the patient remains perfectly still between the two scans. Any patient motion in this case results in an error in the implicitly registered simulation CT and ultrasound, leading to an error in the localisation of the target in the treatment room.

\section{METHODS}

In order to evaluate the accuracy of the proposed ultrasound target localisation system, the entire process of radiation therapy using our system, from simulation to irradiation, was tested on the phantom shown in Fig. 2. This phantom contains a formulation of radiosensitive dosimeter gel developed for verifying 3D dose distributions in radiotherapy which is a 'normoxic' (fabricated under normal atmospheric conditions) variant of the type used by Hilts et al. ${ }^{10}$ Once the phantom was correctly positioned on the LINAC couch using the proposed localisation system, it was irradiated with a pre-planned dose. The location of the actual delivered dose can then be determined from the gel response through subsequent CT scanning and compared to the planned dose. Detailed descriptions of various steps in the experiment protocol are given below. 


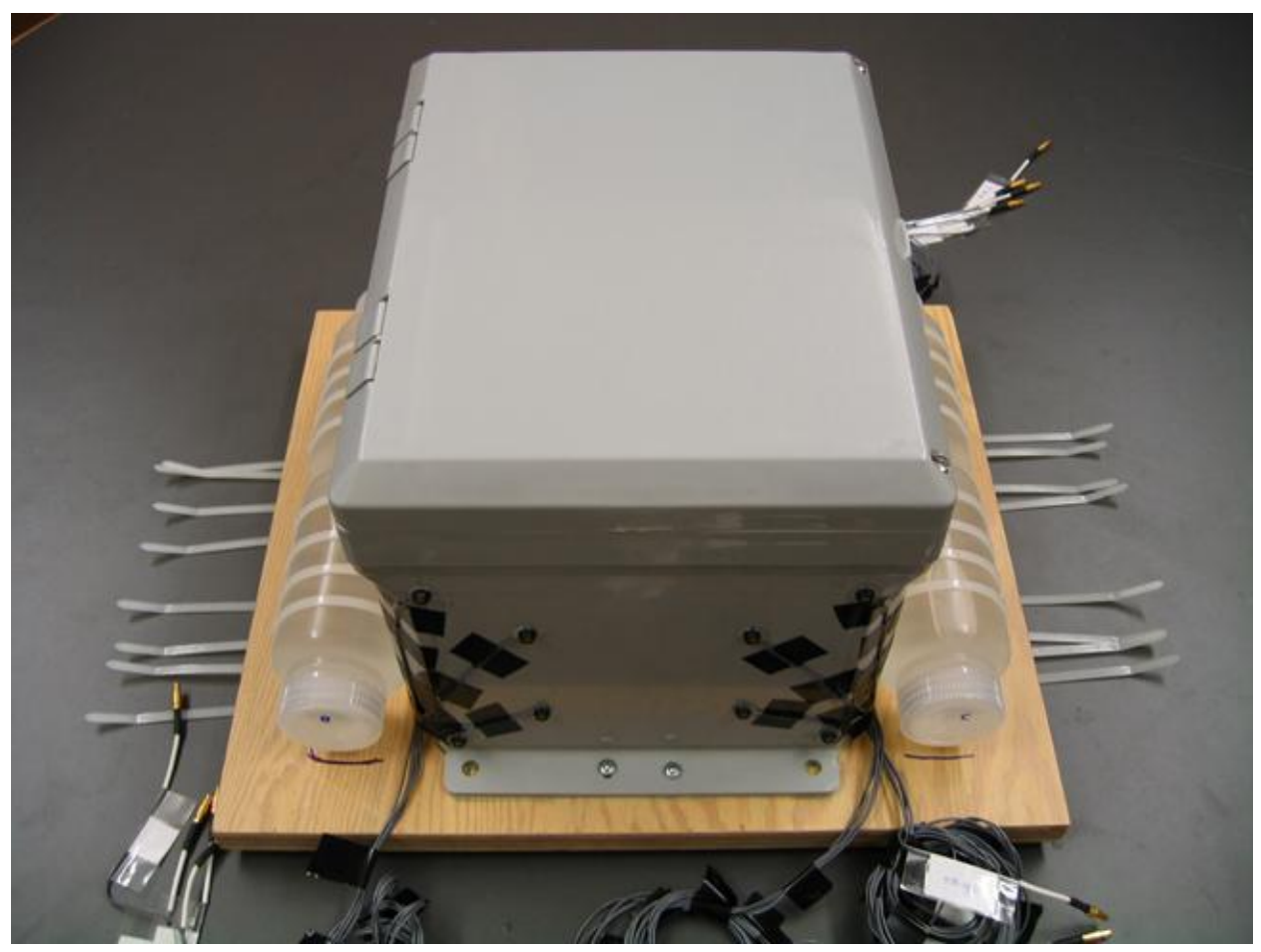

Figure 2. Phantom used to test localisation system accuracy. The large box contains an ultrasound tissue equivalent material $^{11}$ and 3 embedded ballons filled with the same material plus a high concentration of cellulose to act as hyperechogenic features in ultrasound. The lid of the box can be opened to allow access by the ultrasound probe. The four polypropylene NALGENE ${ }^{\mathrm{R}}$ ) bottles contain the dosimeter gel and are rigidly secured to the wooden base with cable ties. IRLEDs are taped to the side wall of the box.

\subsection{Simulation Scans}

For convenience, the simulation ultrasound scan of the phantom was acquired first in our lab using the GE Voluson 730 Expert ultrasound (GE Healthcare, Waukesha, WI) with the 3D RAB4-8P probe while both the probe and phantom were tracked simultaneously by OPTOTRAK. The ultrasound probe calibration matrix $\mathbf{U}_{\mathbf{T}_{\mathbf{P}}}$ was determined by imaging a cross-wire calibration phantom. ${ }^{12}$

The phantom was then transported to the Vancouver Center of the British Columbia Cancer Agency (BCCA) and scanned using a PQ5000 CT simulator (Picker International, Cleveland, OH) with a slice thickness and distance of $3 \mathrm{~mm}$, a pixel spacing of $0.94 \mathrm{~mm}$, scan time of $1 \mathrm{~s}$, tube voltage of $130 \mathrm{kV}$, and tube current of $200 \mathrm{~mA}$.

To register the simulation ultrasound and CT images, the series of transformations outlined in Fig. 1 are used. The phantom IRLED locations were extracted from the CT scan by finding their image centroid and applying a calibrated offset to account for the IRLED offset inside its plastic housing. The transformation ${ }^{\text {Pat }} \mathbf{T}_{\mathbf{C T}}$ was calculated using a least-squares point-based registration algorithm. ${ }^{13}$

\subsection{Treatment Planning}

Treatment planning was performed on the Eclipse system (Varian Medical Systems, Palo Alto, CA). Four isocenters were selected, each near the middle of one of the four dosimeter gel bottles. Each isocenter was given the three field dose plan shown in Fig. 3. The $6 M V$ beam from the Varian 21EX LINAC (Varian Medical Systems, Palo Alto, CA) was chosen to deliver the radiation with square fields $30 \mathrm{~mm}$ wide. The isocenters were irradiated to a maximum dose of $15 G y$. 


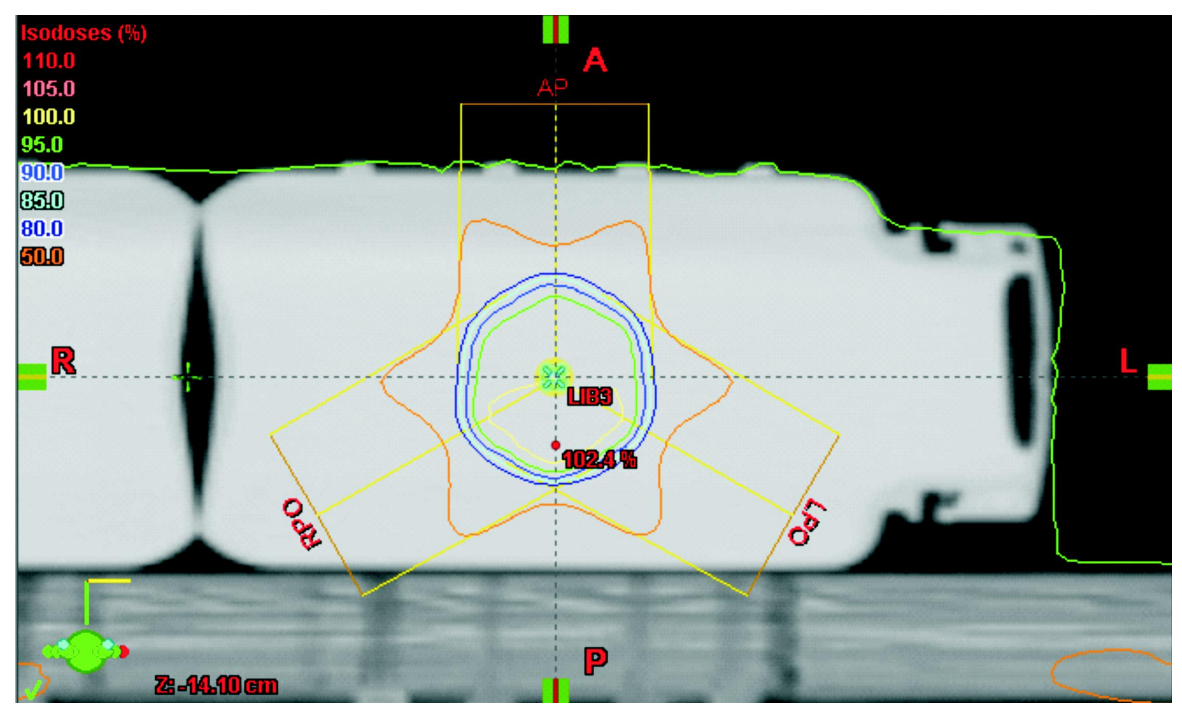

Figure 3. Field arrangement and isodose contours at one of the selected isocenters.

\subsection{Irradiation}

The dosimeter gel's sensitivity to radiation declines with age, so fresh bottles of gel were made approximately 6 hours prior to irradiation. They were strapped onto the base of the phantom in approximately the same positions they were during simulation. The OPTOTRAK was positioned inside the treatment unit against the wall at the inferior end of the couch. To calibrate OPTOTRAK to the LINAC machine isocenter (the transformation ${ }^{\mathbf{L}} \mathbf{T}_{\mathbf{O}^{\prime}}$ in Fig. 1), the patient alignment lasers were used. The lateral and overhead laser crosshair locations on the sides of a box positioned on the couch was touched by a stylus while OPTOTRAK measured its tip location using IRLEDs attached to the stylus base. By shifting the couch location, 10 different points along the left-right and anterior-posterior axis of the LINAC coordinate system were sampled. These points were projected onto the least squares fit plane and the best fit orthogonal axis, along with the origin, were solved using constrained least squares.

For each planned isocenter, the phantom was positioned in the treatment position using lasers and set-up markings. Set-up error was then simulated by moving the couch. The lid of the box on the phantom was then opened and a treatment ultrasound taken while tracking the probe (pre-calibrated to find the value of $\mathbf{P}^{\prime} \mathbf{T}_{\mathbf{U}^{\prime}}$ ) with OPTOTRAK. Next, the treatment ultrasound was registered to the simulation ultrasound on a PC using the Amira 3D visualisation toolkit (TGS Inc., San Diego, CA), which utilises a gradient descent technique to maximise the image correlation coefficient. This is the only remaining coordinate transformation required (see Fig. 1) to determine the location of the planned isocenter in LINAC coordinates. Once this is calculated, the couch shift necessary to bring the planned isocenter in alignment with the LINAC origin (machine isocenter) can be found. This shift is implemented using manual controls to position the motor-driven couch before the phantom is finally irradiated.

\subsection{Gel Response Readout}

Radiation causes the dosimeter gel material to undergo a density change. ${ }^{14}$ This can be detected by CT as a change in the material's Hounsfield units (HU). To extract dose information from the gel, a CT imaging protocol following the guidelines set in Ref. 10 was used. The phantom was scanned using the PQ5000 CT simulator with the exact same parameters as the simulation scan. The high dose regions, in this case the bottles, were scanned repeatedly 16 times to enable image averaging to be performed in order to improve the low signal to noise ratio (this was also done for the simulation $\mathrm{CT}$ ). The simulation and post-irradiation CT scans were then registered using IRLED locations in both. Finally, the averaged simulation scan of the high dose regions was subtracted from the averaged post-irradiation scan to form a difference image indicating changes in Hounsfield units. 


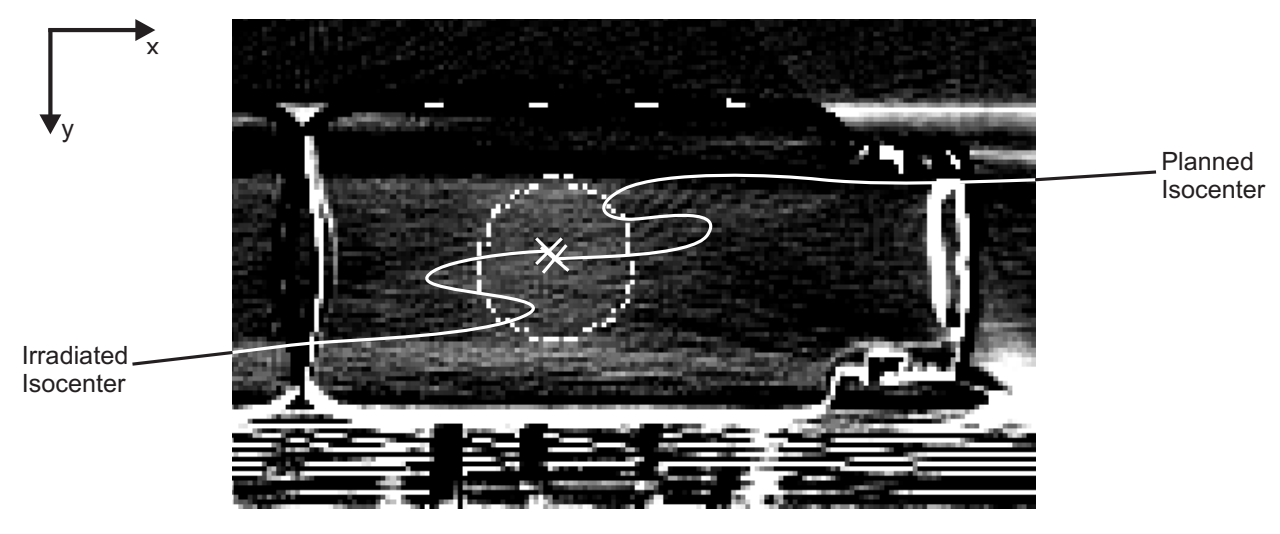

Figure 4. An axial slice of the difference image showing changes in dosimeter gel Hounsfield units after irradiation. The data is windowed from 0 to $30 \mathrm{HU}$. Superimposed on top of the image is the planned $80 \%$ isodose contour (white outline). The planned isocenter and the calculated centroid of the bright pixel cluster (irradiated isocenter) are also marked by crosses.

\section{RESULTS}

The difference image near one of the irradiated bottles is shown in Fig. 4, and is representative of the results obtained. There are several features of note in this image. The bright pixels around the edges of the bottles indicate that there is residual misalignment in their positions after the simulation and post-irradiation CT scans have been registered. This is because the bottles were removed from the phantom after the simulation CT, filled with fresh gel, and replaced only in approximately the same position before irradiation. This slight misalignment should have no effect on the outcome of the experiment, as the isocenter positions are defined in a consistent manner with respect to the IRLEDs on a rigid, permanent part of the phantom. Another feature is the presence of artefacts as bright and dark horizontal bands emanating from the bottleneck and obscuring part of the gel response. These artefacts can be attributed to the unsymmetric shape of the bottles within the scanner bore. Finally, the central bright region inside the bottle corresponds to the location where the gel received the highest dose. It can be confirmed visually that the overlayed planned $80 \%$ isodose contour corresponds closely to the received dose.

To compare the gel response and treatment plan quantitatively, the image weighted centroid of the bright pixel region was calculated as the 'irradiated isocenter'. The band artefacts in the image were removed using edge detection methods and were excluded from the centroid calculation. The difference in locations of the planned isocenter and irradiated isocenter for all four bottles are summarised in Table 1.

Table 1. Summary of phantom positioning results using the proposed ultrasound localisation system. The vector and magnitude difference in the planned isocenter and irradiated isocenter are given in the LINAC coordinate system.

\begin{tabular}{|c||r|r|r|c|}
\hline \multirow{2}{*}{ Bottle } & \multicolumn{3}{|c|}{ Error Vector $(\mathrm{mm})$} & \multirow{2}{*}{\begin{tabular}{c} 
Error \\
\cline { 2 - 4 }
\end{tabular}} \\
$\mathrm{x}$ & $\mathrm{y}$ & $\mathrm{z}$ & Magnitude $(\mathrm{mm})$ \\
\hline \hline 1 & -0.2 & 1.1 & -3.1 & 3.3 \\
\hline 2 & 1.8 & -3.3 & -2.5 & 4.5 \\
\hline 3 & 0.1 & 4.6 & -1.1 & 4.7 \\
\hline 4 & 1.3 & 1.6 & 0.6 & 2.2 \\
\hline
\end{tabular}

The 'simulated error' shown in Table 2 can be subtracted from the 'correction shifts' in the same table to find the difference in phantom position that would result from using the patient alignment lasers and set-up marks instead of 3D ultrasound localisation to position the phantom. This difference can in turn be subtracted from 
the error vector of the 3D ultasound localisation system in Table 1 to derive the positioning error that would be expected from using the patient alignment lasers alone. These results are shown in Table 3.

Table 2. The couch shift used to simulate a set-up error after the phantom is positioned using patient alignment lasers is given as the 'simulated error'. The couch shift used to move the phantom back to its correct position as determined by $3 \mathrm{D}$ ultrasound localisation is shown as the 'correction shift'. All vectors are given in the LINAC coordinate system. Note that the couch moves in one millimeter increments.

\begin{tabular}{|c||r|r|r||r|r|r|}
\hline \multirow{2}{*}{ Bottle } & \multicolumn{4}{c||}{ Simulated Error $(\mathrm{mm})$} & \multicolumn{3}{|c|}{ Correction Shift $(\mathrm{mm})$} \\
\cline { 2 - 7 } & $\mathrm{x}$ & $\mathrm{y}$ & $\mathrm{z}$ & $\mathrm{x}$ & $\mathrm{y}$ & $\mathrm{z}$ \\
\hline \hline 1 & 5 & 0 & 0 & -6 & 0 & -1 \\
\hline 2 & 0 & 0 & 5 & 0 & 1 & -7 \\
\hline 3 & 0 & 5 & 0 & -2 & -7 & -2 \\
\hline 4 & 5 & 5 & 5 & -5 & -5 & -8 \\
\hline
\end{tabular}

Table 3. Summary of phantom positioning results using the patient alignment lasers. The vector and magnitude difference in the planned isocenter and irradiated isocenter are given in the LINAC coordinate system.

\begin{tabular}{|c||r|r|r|c|}
\hline \multirow{2}{*}{ Bottle } & \multicolumn{3}{|c|}{ Error Vector $(\mathrm{mm})$} & \multirow{2}{*}{\begin{tabular}{c} 
Error \\
\cline { 2 - 4 }
\end{tabular}} \\
$\mathrm{x}$ & $\mathrm{y}$ & $\mathrm{z}$ & Magnitude $(\mathrm{mm})$ \\
\hline \hline 1 & 0.8 & 1.2 & -2.1 & 2.6 \\
\hline 2 & 1.8 & -4.3 & -0.4 & 4.7 \\
\hline 3 & 2.1 & 6.6 & 1.0 & 7.0 \\
\hline 4 & 1.3 & 1.6 & 3.6 & 4.1 \\
\hline
\end{tabular}

\section{DISCUSSION}

The positioning errors given in Table 3 actually represent a combination of the error in phantom positioning using the patient alignment lasers, as well as the error in determining the location of the irradiated isocenter from the dosimeter gel. The errors from reading the dosimeter gel response are additional inaccuracies introduced by our experiment, and will not be present in clinical practice. Therefore, the accuracy of laser positioning in practice is expected to be better than the values shown in Table 3. However, one must also keep in mind that positioning a phantom is still a highly idealised situation. Organ motion and skin mark migration in real patients will likely introduce other errors in positioning using lasers.

Similarly, the positioning errors given in Table 1 represent a combination of the phantom positioning error using 3D ultrasound localisation, error from reading the dosimeter gel response, and also error from couch movement. Since the couch position could only be specified with a finite resolution, shifts determined by our ultrasound system could only be implemented to the nearest millimeter. From a clinical perspective, the most significant sources of errors in our 3D ultrasound localisation system include simulation CT and ultrasound registration, OPTOTRAK to LINAC calibration, ultrasound probe calibration, and 3D ultrasound-to-ultrasound registration.

Based on previous phantom studies, our method for registering simulation CT and ultrasound scans (including IRLED detection in the CT scan) can be expected to achieve a RMS registration error of $1.67 \mathrm{~mm}$. Currently, the accuracy of our method of calibrating the OPTOTRAK to the LINAC machine isocenter has not been evaluated. However, the limiting factors in this procedure are the accuracy of the room lasers (lasers to machine isocenter transformation), which has a quality assurance tolerance of $2 \mathrm{~mm}$ in each axis, and OPTOTRAK to laser transformation accuracy, which is limited by the width of the laser beam (approximately 0.5mm). Our technique of ultrasound probe calibration has been found to have a RMS point accuracy error of $2.15 \mathrm{~mm} .^{12}$ 
Our method of automatic ultrasound-to-ultrasound image registration using the image correlation coefficient has previously been tested on a cross-wire phantom. The image registration transformations were compared to OPTOTRAK measurements of the ultrasound probe positions during image acquisition, and a registration accuracy within the order of the pixel size, $0.6 \mathrm{~mm}$, was achieved. This, as well as results obtained using the phantom in this experiment, represents the best case scenario for image registration as there were many clear features present in the ultrasound image. In addition, artefacts such as refraction and speed of sound error were not present. All the registration results for this experiment were visually inspected and no obvious misalignment were detected. The effect of any mis-registration on the accuracy of our results, however, are amplified by the lever-arm effect as the localisation targets are not directly in the ultrasound image field of view. Recall that the cylinders of dosimetric gels are adjacent to the box used for ultrasound scanning. The mean distance from the middle of the simulation ultrasound volume to the four localisation targets is $160 \mathrm{~mm}$. Even with a registration error of 1 degree inside the ultrasound volume, the misalignment at this distance away would be $2.8 \mathrm{~mm}$. This means the reported errors for our system represent the worst case scenario in terms of geometry.

The limiting factor on the accuracy of the dosimeter gel is the CT scanning parameters used to image the gel response. As it can be seen from the results shown, a change of only approximately $10 \mathrm{HU}$ can be expected from the irradiated gel. The scan parameters used in this experiment could be further optimised in accordance to Hilts et $\mathrm{al}^{10}$ to improve the image signal to noise ratio. In addition, the presence of $\mathrm{CT}$ artefacts have obscured dose information in the vertical direction of our phantom. This could be the cause of the relatively large 'positioning' errors in the $y$ direction shown in Tables 1 and 3. Similarly, the poorer spatial resolution in the CT slice direction may cause greater uncertainties in the $z$ direction of the positioning error determined from our experiments. A slice spacing of $3 \mathrm{~mm}$ was chosen to balance between trade-offs in image noise, scan time, and spatial resolution. Thus, the $x$ component of the error is likely the best indicator of phantom position accuracy. As seen from Table 1, the system has an overall accuracy of less than $2 \mathrm{~mm}$ in positioning the phantom in this direction.

Comparing the positioning error of the 3D ultrasound localisation system to that of the room lasers, it can be seen that ultrasound localisation can achieve an accuracy better than the traditional method of patient set-up. For real patients, the expected set-up error using our ultrasound localisation system will be less than the values shown in Table 1, as the errors introduced by the validation procedure in this experiment will not be included. However, registering ultrasound images of real patient anatomy may introduce additional errors due to poorer image quality and the presence of non-reproducible ultrasound imaging artefacts which cannot be cancelled.

\section{CONCLUSIONS}

Compared to other similar research done on quality assurance for ultrasound target localisation in radiotherapy, ${ }^{7}$ our work is not confined to the use of ultrasound to measure simulated phantom movement. Instead, we have attempted to position a phantom like a real patient in the treatment room with respect to an absolute frame of reference (that of the LINAC machine isocenter). Further, the phantom is irradiated and the changes in its material properties are compared with the treatment plan to verify the accuracy of the ultrasound positioning system. Thus, the entire process of radiotherapy treatment using our proposed techniques, including simulation imaging, treatment planning, patient positioning at the time of treatment, and irradiation, has been simulated. Our general validation methodology is applicable to any other image-guided patient positioning system for radiotherapy.

The preliminary results from our experiments have been encouraging and suggest that patient positioning using our proposed methods is able to achieve an overall accuracy of less than $5 \mathrm{~mm}$, and is no worse than the current standard of using room lasers to set-up patients.

Although we have tested our system on a phantom, real patients offer a number of challenges that cannot be simulated. Poor ultrasound image quality in certain anatomical areas could make the automatic registration of such images unfeasible. More sophisticated registration algorithms may also have to be used to deal with non-rigid organ motion. How well our proposed system copes with ultrasound imaging artefacts such as organ deformation from probe pressure in practice compared to other existing ultrasound localisation systems remains to be investigated. These areas will be the focus of future research. 


\section{ACKNOWLEDGMENTS}

This research was funded by the Natural Sciences and Engineering Research Council of Canada (NSERC). Generous support was given to carry out the experimental aspects of this project by Dr. Cheryl Duzenli and Dr. Susan Zhang of the Medical Physics department at the British Columbia Cancer Agency. Assistance in producing the dosimeter gel was provided by Dr. Andrew Jirasek, Department of Physics and Astronomy, University of Victoria.

\section{REFERENCES}

1. K. M. Langen and D. T. L. Jones, "Organ motion and its management," Int. J. Radiat. Oncol. Biol. Phys. 50(1), pp. 265-278, 2001.

2. J. Lattanzi, S. McNeeley, W. Pinover, E. Horwitz, I. Das, T. E. Schultheiss, and G. E. Hanks, "A comparison of daily ct localisation to a daily ultrasound-based system in prostate cancer," Int. J. Radiat. Oncol. Biol. Phys. 43(4), pp. 719-725, 1999.

3. C. F. Serago, S. J. Chungbin, S. J. Buskirk, G. A. Ezzell, A. C. Collie, and S. A. Vora, "Initial experience with ultrasound localisation for positioning prostate cancer patients for external beam radiotherapy," Int. J. Radiat. Oncol. Biol. Phys. 53(5), pp. 1130-1138, 2002.

4. X. Artignan, M. Smitsmans, J. Lebesque, D. Jaffray, M. van Her, and H. Bartelink, "Online ultrasound image guidance for radiotherapy of prostate cancer: impact of image acquisition on prostate displacement," Int. J. Radiat. Oncol. Biol. Phys. 59(2), pp. 595-601, 2004.

5. K. M. Langen, J. Pouliot, C. Anezinos, M. Aubin, A. R. Gottschalk, I.-C. Hsu, D. Lowther, Y.-M. Liu, K. Shinohara, L. J. Verhey, V. Weinberg, and M. Roach III, "Evaluation of ultrasound-based prostate localisation for image-guided radiotherapy," Int. J. Radiat. Oncol. Biol. Phys. 57(3), pp. 635-644, 2003.

6. S. L. Meeks, J. M. Buatti, L. G. Bouchet, F. J. Bova, T. C. Ryken, E. C. Pennington, K. M. Anderson, and W. A. Friedman, "Ultrasound-guided extracranial radiosurgery: technique and application," Int. J. Radiat. Oncol. Biol. Phys. 55(4), pp. 1092-1101, 2003.

7. W. A. Tomé, S. L. Meeks, N. P. Orton, L. G. Bouchet, and F. J. Bova, "Commissioning and quality assurance of an optically guided three-dimensional ultrasound target localization system for radiotherapy," Med. Phys. 29(8), pp. 1781-1788, 2002.

8. F. Cury, G. Shenouda, L. Souhami, M. Duclos, S. Faria, M. David, R. Corns, and T. Falco, "Comparison of bat system and a new 3d trans-abdominal ultrasound-based image-guided system for prostate daily localization during external beam radiotherapy," in 46th ASTRO Annual Meeting, 2003.

9. J. P. McGahan, J. Ryu, and M. Fogata, "Ultrasound probe pressure as a source of error in prostate localization for external beam radiotherapy," Int. J. Radiat. Oncol. Biol. Phys. 60(3), pp. 788-793, 2004.

10. M. Hilts, A. Jirasek, and C. Duzenli, "Technical considerations for implementation of x-ray ct polymer gel dosimetry," Phys. Med. Biol. 50, pp. 1727-1745, 2005.

11. M. M. Burlew, E. L. Madsen, J. A. Zagzebski, R. A. Banjavic, and S. W. Sum, "A new ultrasound tissueequivalent material," Radiology 134, pp. 517-520, 1980.

12. T. C. Poon and R. N. Rohling, "Comparison of calibration methods for spatial tracking of a 3-d ultrasound probe," Ultrasound Med. Biol. 31(8), pp. 1095-1108, 2005.

13. B. K. P. Horn, "Closed-form solution of absolute orientation using unit quaternions," J. Opt. Soc. Am. Ser. A. 4(4), pp. 629-642, 1987.

14. J. V. Trapp, G. Michael, Y. de Deene, and C. Baldock, "Attenuation of diagnostic energy photons by polymer gel dosimeters," Phys. Med. Biol. 47(23), pp. 4247-4258, 2002. 